\title{
Locating the Dust in A Star Debris Discs
}

\author{
Mark Booth ${ }^{1,2}$, Grant Kennedy ${ }^{3}$, Bruce Sibthorpe ${ }^{4}$, \\ Brenda C. Matthews ${ }^{2,1}$, Mark C. Wyatt ${ }^{3}$, Gaspard Duchêne ${ }^{5,6}$, \\ J. J. Kavelaars ${ }^{2,1}$, David Rodriguez ${ }^{7}$, Jane S. Greaves ${ }^{8}$, \\ Alice Koning ${ }^{1,2}$, Laura Vican ${ }^{9}$, George H. Rieke ${ }^{10}$, Kate Y. L. Su ${ }^{10}$, \\ Amaya Moro-Martín ${ }^{11}$ and Paul Kalas ${ }^{5,12}$ \\ ${ }^{1}$ Dept. of Physics \& Astronomy, University of Victoria, Elliott Building, 3800 Finnerty Rd, \\ Victoria, BC, V8P 5C2 Canada; email: markbooth@cantab.net \\ ${ }^{2}$ NRC of Canada, 5071 West Saanich Road, Victoria, BC, V9E 2E7 Canada \\ ${ }^{3}$ Institute of Astronomy, Madingley Rd, Cambridge CB3 0HA, UK \\ ${ }^{4}$ UK ATC, Royal Observatory, Blackford Hill, Edinburgh EH9 3HJ, UK \\ ${ }^{5}$ Astronomy Department, UCB, Hearst Field Annex B-20, Berkeley, CA 94720-3411, USA \\ ${ }^{6}$ UJF-Grenoble 1 / CNRS-INSU, IPAG UMR 5274, Grenoble, F-38041, France \\ ${ }^{7}$ Universidad de Chile, Camino el Observatorio 1515, Las Condes, Santiago, Chile \\ ${ }^{8}$ School of Physics \& Astronomy, University of St Andrews, North Haugh, St Andrews, Fife \\ KY16 9SS, UK \\ ${ }^{9}$ Dept. of Physics \& Astronomy, UCLA, 475 Portola Plaza, Los Angeles, CA 90095-1547, USA \\ ${ }^{10}$ Steward Observatory, University of Arizona 933 N Cherry Avenue Tucson, AZ 85721 \\ ${ }^{11}$ Centro de Astrobiología (CSIC-INTA), 28850 Torrejón de Ardoz, Madrid, Spain \\ ${ }^{12}$ SETI Institute, 515 North Whisman Rd., Mountain View, CA 94043, USA
}

\begin{abstract}
Using photometry at just two wavelengths it is possible to fit a blackbody to the spectrum of infrared excess that is the signature of a debris disc. From this the location of the dust can be inferred. However, it is well known that dust in debris discs is not a perfect blackbody. By resolving debris discs we can find the actual location of the dust and compare this to that inferred from the blackbody fit. Using the Herschel Space Observatory we resolved many systems as part of the DEBRIS survey. Here we discuss a sample of 9 discs surrounding A stars and find that the discs are actually located between 1 and 2.5 times further from their star than predicted by blackbody fits to the spectral energy distribution (SED). The variation in this ratio is due to differences in stellar luminosities, location of the dust, size distribution and composition of the dust.
\end{abstract}

Keywords. circumstellar matter, planetary systems, infrared: stars

Debris discs are formed of dust and planetesimals that are the remnants of the planet formation process. By understanding debris discs we can learn about the planet formation process, dynamical history of the planetary system and potentially even discover new planets from asymmetries in the dust (for a review see e.g. Wyatt 2008).

DEBRIS (Disc Emission via a Bias-free Reconnaissance in the Infrared/Submillimetre) was an open time key project (PI: Brenda Matthews) on Herschel. The aim of the project was to conduct an unbiased, flux limited survey of the nearest stars for debris discs (Phillips et al. 2010). The survey (Matthews et al. 2010) has observed 446 systems (of spectral types A, F, G, K and M) at wavelengths of 100 and $160 \mu \mathrm{m}$ with the PACS instrument and many of these systems have also been followed up at $70 \mu \mathrm{m}$ and with SPIRE at 250, 350 and $500 \mu \mathrm{m}$.

In Booth et al. (2013) we present observations of 9 A stars from the DEBRIS sample that host debris discs resolved by Herschel: $\alpha \mathrm{CrB}, \beta$ Uma, $\lambda$ Boo, $\epsilon$ Pav, $\zeta$ Eri, $\gamma$ Tri, $\rho$ Vir, 30 Mon and $\beta$ Tri. We fit a blackbody to the excess spectrum in these systems based on data from the PACS and SPIRE instruments and ancillary data from the 

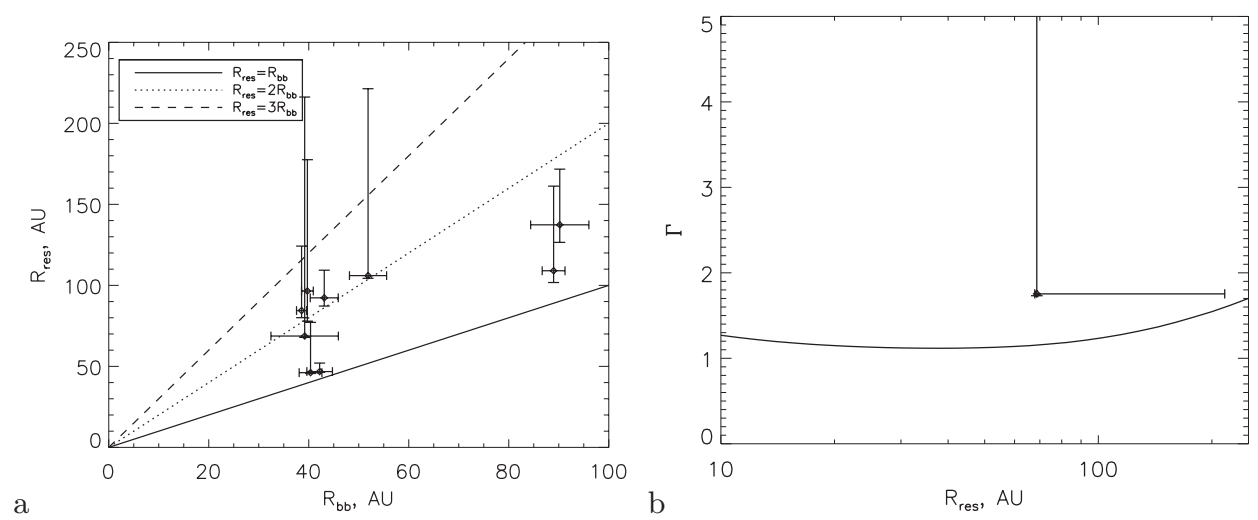

Figure 1. a: Resolved radii versus blackbody radii. The errors show the range of best fits for the three different wavelengths. The lines represent what we would expect for different values of $\Gamma=R_{\text {res }} / R_{b b}$. b: Ratio of radii from the blackbody fitting to resolved radii against the resolved (actual) radii. The line shows the expected ratio variation for stars of spectral type A1. The point shows the observed values for 30 Mon.

literature and infer a radius, $R_{b b}$, from this. To find the actual location of the dust, $R_{\text {res }}$ (the resolved radii), we use a thin-toroid grid-search algorithm. Comparison of these two values is shown in Fig. 1a.

From Fig. 1a we can see that there is some variation in the ratio $R_{r e s} / R_{b b}$ between 1 and 2.5. Some of this variation is simply due to the different spectral types and, therefore, luminosities of the stars. The difference between the radius inferred from the blackbody fit and the actual location of the dust is due to blackbodies being a poor approximation for small grains. Dust grains are inefficient at absorbing and re-emitting wavelengths longer than the size of the grain and so become hotter than blackbodies, producing a spectrum equivalent to blackbodies closer to the star.

By assuming a Dohnanyi (1969) size distribution down to the blowout limit and grains that are composed of a silicate core and organic mantle (Li \& Greenberg 1997) we can simulate what we expect the radius ratio to be. We find that taking account of the stellar luminosity and location of the dust is not enough to explain the radius ratio for some of the systems, meaning that there must be other differences in the grain populations of some systems. For instance, 30 Mon has a higher ratio of $\Gamma=R_{\text {res }} / R_{b b}$ than is expected for its spectral type (Fig. 1b). This could be explained by a larger than expected number of small grains or a different composition to that normally assumed for debris disc dust.

We also find that, although the SEDs are generally well fit by a single temperature blackbody, a number of the images are not well fit by a narrow ring model and require a wide ring or multiple rings to explain the imaged dust emission.

\section{References}

Booth, M., Kennedy, G., Sibthorpe, B., et al. 2013, MNRAS, 428, 1263

Dohnanyi, J. S. 1969, J. Geophys. Res., 74, 2531

Li, A. \& Greenberg, J. M. 1997, A\&A, 323, 566

Matthews, B. C., Sibthorpe, B., Kennedy, G., et al. 2010, A\&\&A, 518, L135

Phillips, N. M., Greaves, J. S., Dent, W. R. F., et al. 2010, MNRAS, 403, 1089

Wyatt, M. C. 2008, ARA\&A, 46, 339 